\title{
STABLE VECTOR BUNDLES OF RANK TWO ON ENRIQUES SURFACES
}

\author{
HOIL KIM
}

\begin{abstract}
We describe the moduli spaces of stable vector bundles of rank 2 on Enriques surfaces. They all have the structure of the fibrations reflecting those of Enriques surfaces.
\end{abstract}

\section{Introduction}

Moduli spaces of stable vector bundles of rank 2 on complex surfaces have been studied by several authors. The structures of the moduli spaces of stable bundles on surfaces such as rational surfaces ([2], [12], [9]), ruled surfaces([5], [24]), K3 surfaces ([15], [16], [21], [22]), elliptic surfaces ([11], [10], [18]) and some surfaces of general type ([4], [8]) have been described. In this paper, we study the moduli spaces of stable vector bundles of rank two on Enriques surfaces. Every Enriques surface is elliptic. So, we can compare our results with those of R. Friedman, who gave a complete description of the moduli spaces of stable vector bundles of rank two with $c_{1}=0$ and $c_{2} \geq 2 p_{g}+2$ on regular elliptic surfaces ([10]). We could describe the moduli spaces of vector bundles of rank two with general Chern classes on Enriques surfaces as two different types (also showing the existences), so that Friedman's result can be included in these two types. They all have the structure of fibrations reflecting the fibration structure of the original Enriques surfaces.

On the other hand, the universal covering space of an Enriques surface is a $K 3$ surface. So, we can compare these moduli spaces with moduli spaces of stable bundles on the corresponding $K 3$ surface. In fact, we showed that the moduli space of stable bundles on an Enriques surface is mapped by degree two onto a Lagrangian subvariety in the moduli space of stable bundles, which is a symplectic variety, on the covering $K 3$ surface [13]. In this paper, we mention the corresponding components

Received January 5, 2005.

2000 Mathematics Subject Classification: 14J28, 14J60.

Key words and phrases: stable vector bundles, Enriques surfaces, moduli spaces. 
of the moduli spaces of stable bundles on the $K 3$ surface. The complete description will be described later.

The contents of this paper is as follows. In chapter one, we will cover the preliminary facts and known results related to our work. In the following two chapters, we state the main results based on this classification and in the final chapter, we will make some remarks.

The author is very grateful to professor Igor Dolgachev for his suggestion of this problem and much helps. This work was also influenced by Prof. S. Mukai's suggestion that on Enriques surfaces we can use the big Picard group to transform the given moduli spaces to some known moduli spaces. He also wants to show thanks to Prof. A. Tyurin and D.Huybrecht for helpful discussions. This work was financially supported by KRF-2003-041-C20009.

\section{Preliminaries}

An Enriques surface is a projective nonsingular surface $X$ with $2 K_{X} \sim$ $0_{\text {s. }}$ (but $K_{X} \nsim 0$ ), where $K_{X}$ is the canonical divisor of $X$ and $H^{1}\left(X, O_{X}\right)$ $=0$. The unramified double covering space of $X$ defined by the torsion class $K_{X}$ is an algebraic $K 3$ surface with a fixed point free involution. Every Enriques surface $X$ admits an elliptic fibration over $\mathbf{P}^{1}$ and every elliptic fibration $f: X \rightarrow \mathbf{P}^{1}$ has exactly two multiple fibres $F_{A}$ and $F_{B}$ such that $2 F_{A}, 2 F_{B}$ are linearly equivalent to a generic fibre $F . K_{X}$ (briefly $K$ ) $\sim F_{A}-F_{B}$. Here $F_{A}$ and $F_{B}$ are called half fibres. The map

$$
c_{1}: \operatorname{Pic} X \rightarrow H^{2}(X, \mathbf{Z})=\mathbf{Z}^{10} \oplus \mathbf{Z}_{2}
$$

is an isomorphism and

$$
\operatorname{Num} X=H^{2}(X, \mathbf{Z}) / \operatorname{Tor}\left(H^{2}(X, \mathbf{Z})\right)=\mathbf{Z}^{10} .
$$

So, we identify $\operatorname{Pic} X$ with $H^{2}(X, \mathbf{Z})$ in this paper. On an Enriques surface or a $K 3$ surface $L^{2}=L \cdot L$ is an even number for any divisor $L$.

On an Enriques surface $X$,

$$
\chi\left(O_{X}(L)\right)=h^{0}(L)-h^{1}(L)+h^{2}(L)=\frac{1}{2} L^{2}+1 .
$$

Definition. An Enriques surface with a smooth rational curve $R\left(R^{2}=-2\right)$ is called a nodal surface, otherwise it is called an unnodal surface. 
REMARK. In the 10 dimensional moduli space of Enriques surfaces, a generic one is unnodal, while the nodal ones form a 9 dimensional subvariety ([6]).

Definition. For any divisor $D>0$, with $D^{2}>0$, we define

$$
\phi(D)=\inf \left\{D \cdot f \mid f \in \operatorname{Num} X, f^{2}=0, f>0\right\} .
$$

Theorem 1. [6] $0<\phi(D)^{2} \leq D^{2}$.

Definition. Let $C$ be an irreducible curve on an Enriques surface $X$ with $C^{2} \geq 2$. Then $|C|$ is called a hyperelliptic linear system if one of the following equivalent conditions is satisfied.

i) The map $\psi_{C}$ associated to $C$ is hyperelliptic, that is, $C^{2}=2$ or it is of degree 2 onto a surface of degree $n-1$ in $\mathbf{P}^{n}$.

ii) $|C|$ has base points.

iii) $\phi(C)=1$.

iv) $C \sim(n-1) E+F$ or $C \sim n E+R$ for some $n$, where $|2 E|$ and $|2 F|$ are elliptic pencils and $R$ is a smooth rational curve such that $E \cdot F=E \cdot R=1$.

Theorem 2. [6] Let $D$ be an effective divisor on an Enriques surface $X$ with $D^{2} \geq 0$. Then

$$
D \sim D^{\prime}+\Sigma m_{i} R_{i}, m_{i} \geq 0,
$$

where $R_{i}$ is a smooth rational curve and one of the following cases occurs:

i) $D^{\prime}$ is an irreducible curve with $D^{\prime 2}>0$;

ii) $D^{\prime}$ is a divisor of canonical type, that is, $D^{\prime} \sim \Sigma n_{i} D_{i}$ is an effective divisor with irreducible components $D_{i}$ such that $K \cdot D_{i}=D^{\prime} \cdot D_{i}=0$ for all $i$;

iii) $D^{\prime} \sim 2 E+R$, where $|2 E|$ is a genus 1 pencil and $R$ is a smooth rational curve with $E \cdot R=1$.

REMARK. [6] There exists $w$, an element of the Weyl group of $X$ generated by the reflections with the smooth rational curves, such that $D^{\prime}=w(D)$. On an unnodal Enriques surface, every effective divisor $D$ with $D^{2}>0$ is ample.

TheOREM 3. [3] Let $D$ be a divisor with $D^{2} \geq 0$ and $D \nsim 0, K$. Then $D$ is effective or $-D$ is effective. If $D$ is effective, then $D+K$ is also effective.

THEOREM 4. [6] For every elliptic pencil $|2 E|$ on an Enriques surface $X$, there exists an elliptic pencil $|2 F|$ such that $E \cdot F=1$. 
Definition. A vector bundle $E$ is called (semi-)stable with respect to an ample divisor $H$, if for any subsheaf $F$, where $0<\operatorname{rank}(F)<$ $\operatorname{rank}(E)$,

$$
\left(c_{1}(F) \cdot H\right) / \operatorname{rank}(F)(\leq)<\left(c_{1}(E) \cdot H\right) / \operatorname{rank}(E) .
$$

REMARK. If the moduli space of stable bundles, $M$, exists, then it is a quasi-projective variety. By the deformation theory, we can compute the dimension of the tangent space $T_{E} M$ at $E \in M$ and the dimension of $M$ at $E$. If the rank $=2$,

$$
\operatorname{dim} T_{E} M=4 c_{2}-c_{1}^{2}-3+h^{2}(\operatorname{End} E),
$$

where $h^{2}(\operatorname{End} E)=0$ if $E \not E(K)$ and 1 if $E \cong E(K)$ and

$$
\operatorname{dim}_{E} M \geq 4 c_{2}-c_{1}^{2}-3 .
$$

THEOREM 5. [13] If $E$ is a singular point in the moduli space of bundles of rank 2 on an Enriques surface $X$, then $E \cong \pi_{*} L$, where $L$ is a line bundle on its covering $K 3$ surface $\bar{X}$ and $\pi$ is a natural projection map from $\bar{X}$ to $X$.

We determined the possible types of Chern classes of rank 2 stable bundles (mod Picard groups) on the Enriques surfaces.

THEOREM 6. [14] we can find a divisor $D(V, n)$ depending only on $V \in \operatorname{Pic} X$ and $n \in \boldsymbol{Z}$ such that for any rank 2 vector bundle $E$ with $c_{1}(E)=V$ and $c_{2}(E)=n$ on an Enriques surface $X$, one of the following holds.

(A) $\frac{1}{2} c_{1}(E(D))^{2}=c_{2}(E(D))-1$,

(B) $\frac{1}{2} c_{1}(E(D))^{2}=c_{2}(E(D))$.

REMARK. In the case (A), we have $2 c_{2} \geq \operatorname{dim} M \geq 2 c_{2}-1$ and in the case (B), we have $2 c_{2}-2 \geq \operatorname{dim} M \geq 2 c_{2}-3$. So, we have only to consider $c_{2} \geq 0$ in the case (A) and $c_{2} \geq 1$ in the case (B). However, in the two main theorems in this paper we assume that $c_{2} \geq 2$ in the case of (A) and $c_{2} \geq 3$ in the case of (B) on unnodal Enriques surfaces. In these cases $c_{1}$ is ample. The cases $c_{2}=0,1$ in (A) and the cases $c_{2}=1,2$ in (B) will be treated differently. We know from Riemann-Roch theorem that any stable bundle $E$ with $c_{1}(E)$ ample satisfying the conditions (A)(or (B)) has a section. Indeed,

$$
\chi(E)=h^{0}(E)-h^{1}(E)+h^{2}(E)=1(2),
$$

so that $h^{0}(E) \geq 1(2)$, since $h^{2}(E)=0$.

Let us fix the notations. 
$X$ is an Enriques surface and its universal covering space, which is a $K 3$ surface, is denoted by $\bar{X}$ and the quotient map from $\bar{X}$ to $X$ is $\pi$. Let $M_{X, H}\left(r, D, c_{2}\right)$ be the moduli space of stable vector bundles with respect to an ample divisor $H$, where $r$ is the rank of the bundle, $D$ is the determinant bundle and $c_{2}$ is the second Chern class. Let $M_{\bar{X}, \pi^{*} H}\left(r, \pi^{*} D, 2 c_{2}\right)$ be the corresponding moduli space of stable bundles with respect to $\pi^{*} H$ on $\bar{X}$. We denote by $i$ the involution on $\bar{X}$ compatible to $\pi$ and by $i^{*}$ the induced involution on $M_{\bar{X}}$.

\section{II. $\frac{1}{2} c_{1}^{2}+1=c_{2}$}

First we state the main theorem of this chapter.

Theorem I. (1) Let $M_{X, H}\left(2, H, c_{2}\right)$ (briefly $M$ ) be the moduli space of stable vector bundles on an unnodal Enriques surface $X$ of rank 2 with determinant $H$, the second Chern class $c_{2}$ which satisfy $\frac{1}{2} H^{2}+1=c_{2} \geq 2$ and are stable with respect to $H$. Then $M$ is nonempty and there is a rational map $f$ from $M$ onto an open set $U$ in $P=\left|H+K_{X}\right|$, the linear system of $H+K_{X}$, where the fiber over a curve $C \in U$ is a nonempty Zariski open subset of the symmetric power of $C, \operatorname{Sym}^{c_{2}}(C)$.

(2) $M$ has at most finitely many isolated singularities and the bundle $E$ corresponding to a singular point is $\pi_{*} L$, where $L$ is a line bundle on the universal covering $K 3$ surface $\bar{X}$ with $L^{2}=-2$.

Proof. First assume that $M$ is non-empty. Then, $\operatorname{dim} M \geq 2 c_{2}-1$. For $E \in M$, there exist an effective divisor $D, I_{Z}$, the ideal sheaf of a zero scheme $Z$ and an exact sequence,

$$
0 \rightarrow O_{X}(D) \rightarrow E \rightarrow I_{Z}(H-D) \rightarrow 0
$$

since $h^{0}(E)>0$. Since $E$ is stable with respect to $H$, we have

$$
D \cdot H<\frac{1}{2} H^{2} .
$$

Here $D \cdot(H-D)+\operatorname{deg} Z=c_{2}$, which implies

$$
D \cdot H \leq D^{2}+c_{2}-\operatorname{deg} Z \text {. }
$$

By the Hodge Index theorem, we obtain

$$
D^{2} H^{2} \leq(D \cdot H)^{2} \leq \frac{1}{2} H^{2} D^{2}+\frac{1}{2} H^{2}\left(c_{2}-\operatorname{deg} Z\right) .
$$


(Note that this implies $D^{2} H^{2} \leq \frac{1}{2} D^{2} H^{2}+\frac{1}{2} H^{2} c_{2}$, so that $D^{2} \leq c_{2}$.) Now we can divide into two cases;

$$
\text { i) } \operatorname{deg} Z \geq c_{2} \text {, ii) } \operatorname{deg} Z<c_{2} .
$$

i) Case $1 ; \operatorname{deg} Z \geq c_{2}$;

In this case we get

$$
D^{2} H^{2} \leq(D \cdot H)^{2} \leq \frac{1}{2} D^{2} H^{2} .
$$

The only possibility is that

$$
D^{2}=0, D \cdot H=0 .
$$

Here $H$ is ample and $D$ is effective. So,

$$
D=0, \operatorname{deg} Z=c_{2} .
$$

Let $M_{0} \subset M$ be the collection of bundles which has an isolated zero section and let

$$
M_{0}^{i}=\left\{E \in M_{0} \mid h^{0}(E(K))=i+1\right\} .
$$

Then $M_{0}^{i}$ is empty for $i<0$, since $h^{0}(E(K)) \geq 1$ for any $E \in M$. From the exact sequence,

$$
0 \rightarrow O_{X}(K) \rightarrow E(K) \rightarrow I_{Z}(H+K) \rightarrow 0,
$$

we have

$$
h^{0}(E(K))=h^{0}\left(I_{Z}(H+K)\right)=h^{1}\left(I_{Z}(H+K)\right)=i+1,
$$

since $h^{0}(K)=h^{1}(K)=\chi\left(I_{Z}(H+K)\right)=h^{2}\left(I_{Z}(H+K)\right)=0$. We compute the dimension of $M_{0}^{i}$ for $i>0$. Let $S^{i}=\left\{Z \mid h^{0}\left(I_{Z}(H+K)\right)=\right.$ $i+1\} \subset \operatorname{Sym}^{c_{2}}(X)$. To every bundle $E \in M_{0}^{i}$ we can associate a cycle $Z \in S^{i}$ and $E$ corresponds to a non-trivial element in

$$
\operatorname{Ext}\left(I_{Z}(H), O_{X}\right) \cong \operatorname{Ext}\left(I_{Z}(H+K), O_{X}(K)\right) \cong H^{1}\left(I_{Z}(H+K)\right)^{*} .
$$

So, we have

$$
\operatorname{dim} M_{0}^{i} \leq \operatorname{dim} S^{i}+i
$$

Now we compute the dimension of $S^{i}(i>0)$. Let $G=\operatorname{Grass}\left(2, h^{0}(H+\right.$ $K)$ ) and let

$$
S=\left\{(V, Z) \mid V \subset H^{0}\left(I_{Z}(H+K)\right)\right\} \subset G \times \operatorname{Sym}^{c_{2}}(X) .
$$

Then, $\operatorname{dim} S=\operatorname{dim} G$ and we have the second projection map

$$
f: S \rightarrow \operatorname{Sym}^{c_{2}}(X) .
$$


The image is the union of $S^{i}(i>0)$ and $f^{-1}(Z)=\left\{(V, Z) \mid V \subset H^{0}\left(I_{Z}(H\right.\right.$ $+K))\}$ for $Z \in S^{i}$. So, we have

$$
\operatorname{dim} S^{i} \leq \operatorname{dim} G-\operatorname{dim} \operatorname{Grass}(2, i+1)=2 c_{2}-2-2 i,
$$

since $\chi(H+K)=h^{0}(H+K)=c_{2}$ for an ample divisor $H$. This implies that

$$
\operatorname{dim} M_{0}^{i} \leq 2 c_{2}-2-i<2 c_{2}-1 \leq \operatorname{dim} M,
$$

if $i>0$.

ii) Case $2 ; \operatorname{deg} Z<c_{2}$;

In this case $D>0$, so that we obtain an exact sequence with a zero scheme Z,

$$
0 \rightarrow O_{X} \rightarrow E(-D) \rightarrow I_{Z}(H-2 D) \rightarrow 0 .
$$

Note that $H \cdot D-D^{2}>0$ and $\operatorname{deg} Z=c_{2}-\left(H \cdot D-D^{2}\right)$.

$$
\text { From } \chi\left(I_{Z}(H-2 D+K)\right)=-\left(H \cdot D-D^{2}\right)<0 \text {, we have }
$$

$$
\begin{aligned}
\operatorname{dim} \operatorname{Ext}\left(O_{X}, I_{Z}\left(H_{2} D\right)\right) & =h^{1}\left(I_{Z}(H-2 D+K)\right) \\
& =h^{0}\left(I_{Z}(H-2 D+K)\right)+\left(H \cdot D-D^{2}\right) \\
& =h^{0}(E(-D+K))+H \cdot D-D^{2}
\end{aligned}
$$

since $h^{2}\left(I_{Z}(H-2 D+K)\right)=h^{2}\left(O_{X}(H-2 D+K)\right)=h^{0}\left(O_{X}(2 D-H)\right)=0$ due to $2 D \cdot H<H^{2}$. Let $M_{D} \subset M$ be the collection of the bundles $E$, where $E(-D)$ has an isolated zero section and let

$$
M_{D}^{i}=\left\{E \in M_{D} \mid h^{0}(E(-D+K))=i\right\} .
$$

Then

$$
\begin{aligned}
\operatorname{dim} M_{D}^{0} & \leq \operatorname{dim} \operatorname{Sym}^{c_{2}-\left(H \cdot D-D^{2}\right)}(X)+H \cdot D-D^{2}-1 \\
& \leq 2\left(c_{2}-\left(H \cdot D-D^{2}\right)\right)+\left(H \cdot D-D^{2}-1\right) \\
& <2 c_{2}-1 \leq \operatorname{dim} M .
\end{aligned}
$$

Now we want to have a bound of $h^{0}(H-2 D+K)$. From the exact sequence,

$$
0 \rightarrow O_{X}(-2 D+K) \rightarrow O_{X}(H-2 D+K) \rightarrow O_{C}(H-2 D+K) \rightarrow 0,
$$

where $C$ is a smooth curve in $|H|$, we have

$$
h^{0}\left(O_{X}(H-2 D+K)\right) \leq h^{0}\left(O_{C}(H-2 D+K)\right) \leq c_{2}-H \cdot D,
$$

by the Clifford's theorem.

(Note that 2 genus $(C)-2=H^{2}>C \cdot(H-2 D+K)>0$.) So,

$$
\begin{aligned}
\operatorname{dim} M_{D}^{1} & \leq h^{0}(H-2 D+K)-1+c_{2}-\left(H \cdot D-D^{2}\right)+\left(H \cdot D-D^{2}\right) \\
& \leq 2 c_{2}-1-H \cdot D<2 c_{2}-1 \leq \operatorname{dim} M .
\end{aligned}
$$




$$
\begin{aligned}
\operatorname{dim} M_{D}^{i}(i \geq 2) \leq & 2\left(h^{0}(H-2 D+K)-2\right)-2(i-2) \\
& +\left(H \cdot D-D^{2}\right)+i-1 \\
\leq & 2 c_{2}-1-i-D^{2}<2 c_{2}-1 \leq \operatorname{dim} M
\end{aligned}
$$

with the same argument as before. Note that $D^{2} \geq 0$ for any effective divisor $D$ since any effective divisor is generated by the curves of the arithmetic genus 1 on an unnodal Enriques surface ([6]).

So, $M_{0}$ is open and dense in $M$ and $M_{0}^{0}$ is open and dense in $M_{0}$, so that we conclude that $M_{0}^{0}$ is open and dense in $M$.

Conversely, each zero cycle $Z$ of degree $c_{2}$ consisting of distinct points on a smooth curve $C \in|H+K|$ with $h^{0}\left(I_{Z}(H)\right)=0$ and $h^{0}\left(I_{Z}(H+\right.$ $K))=1$ determines a unique vector bundle $E(Z, C)$,

$$
0 \rightarrow O_{X} \rightarrow E(Z, C) \rightarrow I_{Z}(H) \rightarrow 0,
$$

since $\operatorname{dimExt}\left(I_{Z}(H), O_{X}\right)=1$ (See [21] and [22]). We claim that $E$ is stable. Suppose that $E$ is not stable. Then there exists an exact sequence,

$$
0 \rightarrow O_{X}(L) \rightarrow E \rightarrow I_{W}(H-L) \rightarrow 0,
$$

such that $L \cdot H \geq \frac{1}{2} H^{2}=c_{2}-1$ and $W$ is a 0 -dimensional scheme. So, we have

$$
L \cdot(H-L)=c_{2}-\operatorname{deg} W \leq c_{2},
$$

which implies

$$
L^{2} \geq 0,
$$

so that $L$ is effective. (Note that $L^{2}$ is even.)

From the condition that $h^{0}\left(I_{Z}(H)\right)=0$, we have $h^{0}(E)=1$ and we already know that $E$ has an isolated 0 -section. So, the injectivity of $O_{X}(L) \rightarrow E$ forces $L$ to be 0 . Otherwise, $E$ has no isolated 0 -section. This gives a contradiction.

Let $N$ be $\left\{(Z, C) \mid Z\right.$ is a 0 -cycle of degree $c_{2}$ of distinct points on a smooth curve $C \in|H+K|, h^{0}\left(I_{Z}(H)\right)=0$ and $\left.h^{0}\left(I_{Z}(H+K)\right)=1\right\} \subset$ $\operatorname{Sym}^{c_{2}}(X) \times|H+K|$.

Now we can construct a vector bundle $\Xi$ on $N \times X$ such that $\left.\Xi\right|_{(Z, C)}=$ $E(Z, C)$, exactly following the method of Okonek and Van de Ven [18]. We define $\Gamma=\{((Z, C), x) \mid(Z, C) \in N, x \in X$ and $x \in \operatorname{Supp} Z\} \subset N \times X$ and denote by $I_{\Gamma}$ its ideal sheaf. We define on $N$,

$$
L=\underline{\operatorname{Ext}}_{\pi_{1}}^{1}\left(I_{\Gamma} \otimes \pi_{2}^{*}\left(O_{X}(H)\right), \pi_{2}^{*}\left(O_{X}\right)\right) .
$$


Since $\operatorname{dimExt}{ }^{1}\left(I_{Z}(H), O_{X}\right)=1$ for any $(Z, C) \in N$, we see that $L$ is a line bundle. By [1], [18], there is a spectral sequence,

$$
\begin{aligned}
& H^{p}\left(\underline{\operatorname{Ext}}_{\pi_{1}}^{q}\left(I_{\Gamma} \otimes \pi_{2}^{*}\left(O_{X}(H)\right), \pi_{2}^{*}\left(O_{X}\right) \otimes \pi_{1}^{*}\left(L^{*}\right)\right)\right) \\
\rightarrow & \operatorname{Ext}^{p+q}\left(I_{\Gamma} \otimes \pi_{2}^{*}\left(O_{X}(H)\right), \pi_{2}^{*}\left(O_{X}\right) \otimes \pi_{1}^{*}\left(L^{*}\right)\right) .
\end{aligned}
$$

From this sequence we derive

$$
\begin{aligned}
& \operatorname{Ext}^{1}\left(I_{\Gamma} \otimes \pi_{2}^{*}\left(O_{X}(H)\right), \pi_{2}^{*}\left(O_{X}\right) \otimes \pi_{1}^{*}\left(L^{*}\right)\right) \\
= & H^{0}\left(\underline{\operatorname{Ext}}_{\pi_{1}}^{1}\left(I_{\Gamma} \otimes \pi_{2}^{*}\left(O_{X}(H)\right), \pi_{2}^{*}\left(O_{X}\right) \otimes \pi_{1}^{*}\left(L^{*}\right)\right)\right) \\
= & H^{0}\left(L \otimes L^{*}\right)=H^{0}\left(O_{N}\right) .
\end{aligned}
$$

Here we used the fact $\operatorname{Hom}\left(I_{Z}(H), O_{X}\right)=0$. So, we can associate to $1 \in H^{0}\left(O_{N}\right)(N$ is connected $)$, an extension

$$
0 \rightarrow \pi_{2}^{*}\left(O_{X}\right) \rightarrow \Xi \rightarrow I_{\Gamma} \otimes \pi_{2}^{*}\left(O_{X}(H) \otimes \pi_{1}^{*}(L)\right) \rightarrow 0
$$

on $N \times X$, with $\Xi$ locally free, and $\left.\Xi\right|_{(Z, C)}$ is isomorphic to the bundle $E(Z, C)$. Then by the universal property of a coarse moduli space $M$, the bundle $\Xi$ induces a morphism $g: N \rightarrow M$, such that it is injective and the image is in $M_{0}^{0}$. By the Zariski's main theorem, $N$ is biregular to its image. $M_{0}^{0}-g(N)=M_{1} \cup M_{2}$, where $M_{1}=\left\{E \in M_{0} \mid h^{0}(E) \geq 2\right\}$ and $M_{2}=\left\{E \in M_{0} \mid\right.$ the unique curve $\left|I_{Z}(H+K)\right|$ is singular $\}$, where $E$ is the non-trivial extension of $I_{Z}(H)$ by $O_{X}$. Then, $\operatorname{dim} M_{1} \leq \operatorname{dim}|H|+$ $\operatorname{dim}|H+K|<2 c_{2}-1$ since $h^{0}\left(I_{Z}(H)\right)=h^{0}(E)-1 \geq 1$ and $\operatorname{dim} M_{2} \leq$ $\operatorname{dim}|H+K|-1+c_{2}<2 c_{2}-1$. So, $g(N)$ is open and dense in $M_{0}^{0}$. Since $N$ is of dimension $2 c_{2}-1$, connected and irreducible, $M$ is also of dimension $2 c_{2}-1$, connected and irreducible. (Note that $M_{0}^{0}$ is open and dense in $M$.)

Let $E$ be a singular point in $M$. Then, $E \cong E(K)$ and $E \cong \pi_{*} L$ for some line bundle $L$ on the universal covering space $\bar{X}([20]) . \pi^{*} E=$ $L \oplus i^{*} L$. We can get $2 c_{1}(E)^{2}=2 L^{2}+2 L \cdot i^{*} L, 2 c_{2}(E)=L \cdot i^{*} L$. So, we obtain $L^{2}=c_{1}(E)^{2}-2 c_{2}(E)=-2,\left(L-i^{*} L\right)^{2}=-4 c_{2}-4$ and $L-i^{*} L \in \pi^{*} H^{\perp}$ which is a negative definite lattice. So, there can be only finitely many decompositions of $\pi^{*} c_{1}(E)$ into a direct sum of a divisor $L$ and its involution $i^{*} L$. So, if $c_{2} \geq 2$, then $\operatorname{dim} M$ must be $2 c_{2}-1>0$, since for a generic $E \in M, E \not E E(K)$ and $h^{2}(\operatorname{End} E)=0$, so that $M$ is generically smooth. (In fact, $\operatorname{dim} N=2 c_{2}-1$.)

REMARK 1 . We can see that there is no singular point in $g(N)$. In fact, any $E$ in the image of $N$ in $M_{0}^{0}$ is uniquely determined by an effective zero cycle $Z$ of degree $c_{2}(E)(=$ genus $(C))$ on a smooth curve $C$. Then, $O_{C}(Z+K)$ has an effective divisor $Z^{\prime}$ which determines $E^{\prime}$, since the degree of $Z+K$ on $C$ is $c_{2}=\operatorname{genus}(C)$. Here $\pi^{*}\left(O_{C}(Z)\right)$ 
is linearly equivalent to $\pi^{*}\left(O_{C}(Z+K)\right)$ on $\pi^{-1}(C)$, so that $\pi^{*} E \cong$ $\pi^{*} E^{\prime}([21])$. Then, $E \cong E^{\prime}$ or $E \cong E^{\prime}(K)$, since $h^{0}\left(\pi^{*}\left(E^{*} \otimes E^{\prime}\right)\right)=$ $h^{0}\left(E^{*} \otimes E^{\prime}\right)+h^{0}\left(E^{*} \otimes E^{\prime}(K)\right)$. However $E$ is not isomorphic to $E^{\prime}$, since $O_{C}(Z) \nRightarrow O_{C}(Z+K)$ if genus $(C) \geq 2$. So, we can conclude that $E(K) \cong E^{\prime} \in g(N)$ and $E$ is not a singular point.

REMARK 2. Let $M_{\bar{X}}\left(2, \pi^{*} H, \pi^{*} c_{2}\right)$ be the moduli space of stable bundles of rank 2 with determinant $\pi^{*} H$ and the second Chern class $\pi^{*} c_{2}$ which are stable with respect to $\pi^{*} H$. Then $M_{\bar{X}}$ has a component $\bar{M}$ which has a fibration structure over $\left|\pi^{*} H\right|$, where the fibre over a curve $D$ in $\left|\pi^{*} H\right|$ is the $\operatorname{Jac}(D)$. Then $M_{0}^{0}$ is mapped into $\bar{M}$ by $\pi^{*}$. We can illustrate it by the following diagram

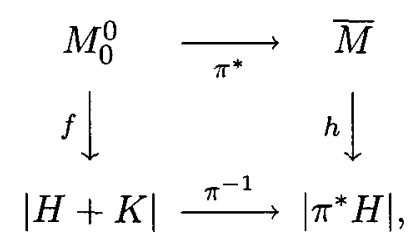

where $f^{-1}(C)=\operatorname{Sym}^{c_{2}}(C)$ for $C \in|H+K|$ and $h^{-1}(D)=\operatorname{Jac}(D)$ for $D \in\left|\pi^{*} H\right|$. Here $\pi^{*}\left(\operatorname{Sym}^{c_{2}}(C)\right)=\operatorname{Jac}\left(\pi^{-1}(C)\right)^{i^{*}}$, the fixed locus in $\operatorname{Jac}\left(\pi^{-1}(C)\right.$ ) by $i^{*}$. (Note that $\operatorname{Sym}^{c_{2}}(C)$ is birational to $\operatorname{Jac}(C)$.) The details will be described in a later paper.

REMARK 3 . In theorem I, we assumed that $c_{1}^{2} \geq 2$. However, $c_{1}^{2} \geq-2$ is necessary for the existence of stable bundles. If $c_{1}^{2}=-2$ and $c_{2}=0$, then there exists a stable bundle if and only if $c_{1}=N+2 S+K$, where $N$ is a nodal class and $S$ is a divisor ([13]). If $c_{1}^{2}=0$, then there exists no stable bundle with $c_{1}=0$ and $c_{2}=1$, but there exists a stable bundle with $c_{1}=f$ and $c_{2}=1$, where $|2 f|$ gives an elliptic system. We will show this as an example.

EXAMPLE. Let $M$ be the moduli space with $c_{1}=F_{A}$ and $c_{2}=1$. Then, we can show easily that $M$ is birational to $F_{B}$ and vice versa following the methods in ([18]).

III. $\frac{1}{2} c_{1}^{2}=c_{2}$

Here we state the main theorem of this chapter.

Theorem II. (1) Let $M_{X, H}\left(2, H, c_{2}\right)$ (briefly $M$ ) be the moduli space of stable vector bundles on an unnodal Enriques surface $X$ of rank 2 with determinant $H$ with the second Chern class $c_{2}$ which satisfy $\frac{1}{2} H^{2}=c_{2} \geq$ 3 and are stable with respect to $H$. Then, $M$ is non-empty. 
(a) If $|H|$ is not hyperelliptic, then there is a rational map $f$ from $M^{\prime}$, a component of $M$, onto an open set $U \in \boldsymbol{P}=|H|$, the linear system of $H$, where the fiber over a curve $C \in U$ is a non-empty open subset of $W_{c_{2}}^{1}(C)$ (special effective divisors of degree $c_{2}$ on $C$ ).

(b) If $|H|$ is hyperelliptic, then $M$ has a structure described in theorem $I$ in chapter $2\left(\frac{1}{2} H^{2}+1=c_{2}\right)$.

(2) $M$ has at most finitely many isolated singularities and the bundle $E$ corresponding to a singular point is $\pi_{*} L$, where $L$ is a line bundle on the universal covering $K 3$ surface $\bar{X}$ with $L^{2}=0$.

Proof. First assume that $M$ is non-empty. Then, $\operatorname{dim} M \geq 2 c_{2}-3$. For $E \in M$ we have $h^{0}(E) \geq 2$. As in the previous case, we have an exact sequence,

$$
0 \rightarrow O_{X}(D) \rightarrow E \rightarrow I_{Z}(H-D) \rightarrow 0,
$$

where $D$ is an effective divisor and $I_{Z}$ is an ideal sheaf of a zero scheme $Z$. In the same way as in the previous proof, we can divide into two cases,

$$
\text { i) } \operatorname{deg} Z \geq c_{2} \text {, ii) } \operatorname{deg} Z<c_{2} \text {. }
$$

i) Case 1: $\operatorname{deg} Z \geq c_{2}$

In this case $D=0$ and $\operatorname{deg} Z=c_{2}$ as we discussed in the proof of the theorem I. Let $M_{0} \subset M$ be the collection of vector bundles which have an isolated zero section and let $M_{0}^{i}=\left\{E \in M_{0} \mid h^{0}(E(K))=i+2\right\}$. Then $M_{0}^{i}$ is non-empty for $i \geq 0$ (empty for $i<0$ ) and for each $E \in M_{0}^{i}$ we have an exact sequence

$$
0 \rightarrow O_{X} \rightarrow E \rightarrow I_{Z}(H) \rightarrow 0
$$

and from this and $\chi\left(I_{Z}(H+K)\right)=1$, we get

$$
h^{0}(E(K))=h^{0}\left(I_{Z}(H+K)\right)=h^{1}\left(I_{Z}(H+K)\right)+1=i+2 .
$$

As in the previous argument,

$$
\begin{aligned}
\operatorname{dim} M_{0}^{i} \leq & \operatorname{dim} \operatorname{Grass}\left(2, h^{0}(H+K)\right)-\operatorname{dim} \operatorname{Grass}\left(2, h^{0}\left(I_{Z}(H+K)\right)\right) \\
& +i-\left(h^{0}(E)-1\right) \\
\leq & 2\left(c_{2}+1-2\right)-2(i+2-2)+i-1=2 c_{2}-3-i .
\end{aligned}
$$

So, $\operatorname{dim} M_{0}^{i}<2 c_{2}-3 \leq \operatorname{dim} M$ for $i>0$.

ii) Case 2: $\operatorname{deg} Z<c_{2}$

In this case $D>0$ and $H \cdot D-D^{2}=c_{2}-\operatorname{deg} Z>0$ and we obtain an exact sequence,

$$
0 \rightarrow O_{X} \rightarrow E(-D) \rightarrow I_{Z}(H-2 D) \rightarrow 0 .
$$


Let $M_{D} \subset M$ be the collection of the bundles $E$, where $E(-D)$ has an isolated zero section. Now we divide into two cases again, a) $\operatorname{deg} Z=c_{2}-1$ and b) $\operatorname{deg} Z<c_{2}-1$.

ii-a) $\operatorname{deg} Z=c_{2}-1$

In this case $H \cdot D-D^{2}=(H-D) \cdot D=1$. We claim that $D$ is a half fiber such that $H \cdot D=1$. So, these cases happen if and only if $|H|$ is hyperelliptic. To prove this claim, first let us compute $(H-D)^{2}$.

$$
(H-D)^{2}=H^{2}-2 H \cdot D+D^{2}>0,
$$

since $H^{2}-2 H \cdot D>0$ and $D^{2} \geq 0$. The first inequality comes from the stability of $E$ and the second one comes from that $D$ is an effective divisor on an unnodal Enriques surface. So, $H-D$ is ample and $D$ is a half fiber, since $D$ is a curve of arithmetic genus 1 . Then, $H \cdot D=$ $(H-D) \cdot D+D^{2}=1$, so that $|H|$ is a hyperelliptic system. Note that

$$
\frac{1}{2} c_{1}^{2}(E(-D))=\frac{1}{2}(H-2 D)^{2}=\operatorname{deg} Z-1=c_{2}(E(-D))-1 .
$$

We know that a generic element in $M_{D}$ is stable with respect to $H^{\prime}=H-$ $2 f$, which is ample, by the result of Qin ([25]). So, we can conclude that $M_{X, H}\left(2, H, c_{2}\right)$ is birational to $M_{X, H^{\prime}}\left(2, H^{\prime}, c_{2}-1\right)$ if $|H|$ is hyperelliptic.

ii-b) $\operatorname{deg} Z<c_{2}-1$

Let $M_{D}^{i}=\left\{E \in M_{D} \mid h^{0}(E(-D+K))=i\right\}$ for $i \geq 0$. Then, for $E \in M_{D}^{i}$ we have

$$
\begin{aligned}
& h^{0}\left(I_{Z}(H-2 D+K)\right)=i, \\
& h^{1}\left(I_{Z}(H-2 D+K)\right)=i-1+\left(H \cdot D-D^{2}\right) .
\end{aligned}
$$

Note that $H \cdot D-D^{2} \geq 2$. As in the previous case,

$$
\begin{aligned}
\operatorname{dim} M_{D}^{0} & \leq 2\left(c_{2}-\left(H \cdot D-D^{2}\right)\right)+\left(H \cdot D-D^{2}-2\right) \\
& =2 c_{2}-\left(H \cdot D-D^{2}\right)-2 \\
& \leq 2 c_{2}-4<\operatorname{dim} M .
\end{aligned}
$$

Since $h^{0}(H-2 D+K) \leq c_{2}+1-H \cdot D$ by the Clifford's theorem as before,

$$
\begin{aligned}
\operatorname{dim} M_{D}^{1} \leq & \left(h^{0}(H-2 D+K)-1\right)+\left(c_{2}-\left(H \cdot D-D^{2}\right)\right) \\
& +\left(H \cdot D-D^{2}-1\right) \\
\leq & c_{2}-(H \cdot D)+c_{2}-1 \\
= & 2 c_{2}-1-(H \cdot D) .
\end{aligned}
$$


If $H \cdot D \geq 3$, then $\operatorname{dim} M_{D}^{1}<\operatorname{dim} M$. Suppose that $H \cdot D=2$. Then $H-2 D$ is ample if $H^{2} \geq 10$, since $(H-2 D)^{2}=10-4 H \cdot D+4 D^{2}>0$, so that $h^{0}(H-2 D+K)=\chi\left(O_{X}(H-2 D+K)\right)=c_{2}-2\left(H \cdot D-D^{2}\right)+1$. So, in this case also, $\operatorname{dim} M_{0}^{1}<\operatorname{dim} M$. If $H^{2}=8$, then $\operatorname{dim} M_{D}^{1}<\operatorname{dim} M$ since $h^{0}(H-2 D+K) \leq 2([6])$. If $H^{2}=4$ or 6 , then $M_{D}^{1}$ is empty, since $h^{0}(H-2 D+K)=0([6])$. For $i \geq 2$, we have

$$
\begin{aligned}
\operatorname{dim} M_{0}^{i} & \leq 2\left(h^{0}(H-2 D+K)-2\right)-2(i-2)+\left(i-2+H \cdot D-D^{2}\right) \\
& \leq 2 c_{2}-i-\left(H \cdot D-D^{2}\right)<\operatorname{dim} M .
\end{aligned}
$$

So, $M_{0}^{0}$ is open and dense in $M$ if $|H|$ is not hyperelliptic and if $|H|$ is hyperelliptic, $M$ is birational to the moduli space in the previous case $\frac{1}{2} c_{1}^{2}+1=c_{2}$.

Conversely, if $Z$ is a divisor on a smooth curve $C \in|H|$ of degree $c_{2}=\operatorname{genus}(C)-1$ with

$$
\begin{gathered}
h^{0}\left(C, O_{C}(Z)\right)=h^{0}\left(C, O_{C}\left(K_{C}-Z\right)\right)=h^{0}\left(I_{Z}(H+K)\right)=2, \\
h^{0}\left(I_{Z}(H)\right)=1,
\end{gathered}
$$

then we can construct a locally free sheaf $E(Z, C)$, uniquely determined by $H^{1}\left(I_{Z}(H+K)\right)$, whose dimension is 1

$$
0 \rightarrow O_{X} \rightarrow E(Z, X) \rightarrow I_{Z}(H) \rightarrow 0,
$$

as in the theorem I. We claim that $E$ is semi-stable and stable for a generic $E$. To prove that $E$ is semi-stable, we can use just the same method that we used in the previous theorem. Suppose $E$ is not stable. Then there exists a divisor $L$ such that $L \cdot H=\frac{1}{2} H^{2}$ and there is an exact sequence,

$$
0 \rightarrow O_{X}(L) \rightarrow E \rightarrow I_{W}(H-L) \rightarrow 0 .
$$

We have

$$
\operatorname{deg} W=c_{2}-H \cdot L+L^{2} \geq 0
$$

so that,

$$
L^{2} \geq 0 \text {. }
$$

However, $L^{2}<2$, not to contradict that $E$ has an isolated zero section. (Note that $h^{0}(E)=2$.) Then,

$$
L^{2}=D^{2}=0, L \cdot D=\frac{1}{2} H^{2}=c_{2}, \operatorname{deg} W=0,
$$

where $D=H-L$. Hence if $E$ is not stable, $E$ is an extension,

$$
0 \rightarrow O_{X}(L) \rightarrow E \rightarrow O_{X}(D) \rightarrow 0,
$$


with the above condition. However there are only finitely many such decompositions $(H=L+D)$, since $H$ is ample and $(L-D) \cdot H=0$, $(L-D)^{2}=-H^{2}$ and the intersection form on $H^{\perp}$ is negative definite. Note that also the dimension of $\operatorname{Ext}^{1}\left(O_{X}(D), O_{X}(L)\right)-1=h^{1}(L-D)-$ $1=c_{2}-2$ which is smaller than $\operatorname{dim}|H|+\rho\left(c_{2}+1,1, c_{2}\right)=2 c_{2}-3$ for $c_{2}>1$, where $\rho(g, r, d)=g-(r+1)(g-d+r)$ is the Brill-Noether number.

If $|H|$ is hyperelliptic, then $E(Z, C)$ is not locally free if $c_{2}>2$. This claim comes from the fact that $E(Z, C)$ is locally free if and only if $|Z|$ is fixed point free on $C$.

Let $N$ be $\left\{(Z, C) \mid Z\right.$ is a 0 -cycle of degree $c_{2}$ on a smooth curve $C \in$ $|H|$ with $h^{0}\left(C, O_{C}(Z)\right)=2$ and $\left.h^{0}\left(I_{Z}(H)\right)=1\right\} \subset \operatorname{Sym}^{c_{2}} X \times|H|$.

Then we can construct a vector bundle $\Xi$ on $N \times X$ such that $\left.\Xi\right|_{(Z, C) \times X}$ $=E(Z, C)$ as in the previous case. By the universal property of the coarse moduli space $M$, the bundle $\Xi$ induces a morphism,

$$
g: N \rightarrow M \text {. }
$$

We will show that $g((Z, C))=g\left(\left(Z^{\prime}, C^{\prime}\right)\right)$ if and only if $C=C^{\prime}$ and $Z$ is linearly equivalent to $Z^{\prime}$ on $C$. First we show that $O_{C}(Z) \cong O_{C}\left(Z^{\prime}\right)$ implies $E(Z, C) \cong E\left(Z^{\prime}, C\right)$. We follow the method of Tyurin exactly [21]. The curve $C \supset Z$ defines a one dimensional space of sections spanned by $s \in H^{0}\left(I_{Z}(C)\right)$ which lifts uniquely to a one-dimensional subspace spanned by $\bar{s} \in H^{0}(E(Z, C))$. Consider the linear span $W=$ $\left\langle H^{1}\left(I_{Z}(H+K), \bar{s}\right\rangle \subset H^{0}(E(Z, C))\right.$ and the canonical homomorphism,

$$
0 \rightarrow W \otimes O_{X} \rightarrow E(Z, C) \rightarrow O_{C}\left(K_{C}-Z\right) \rightarrow 0 .
$$

Dualizing this, we get an exact sequence,

$$
0 \rightarrow E(Z, C)^{*} \rightarrow W^{*} \otimes O_{X} \rightarrow O_{C}(Z) \rightarrow 0
$$

and an isomorphism

$$
W^{*} \cong H^{0}\left(O_{C}(Z)\right)
$$

since $h^{0}\left(E(Z, C)^{*}\right)=h^{1}\left(E(Z, C)^{*}\right)=h^{1}(E(Z, C)(K))=0$. To the hyperplane $H^{1}\left(I_{Z}(H+K)\right) \subset W$ there corresponds the one dimensional space of sections spanned by $s \in H^{0}\left(O_{C}(Z)\right) \cong W^{*}$ such that $(s)_{0}=Z$. Next we consider a section $s^{\prime} \in H^{0}\left(O_{C}(Z)\right)$ such that $\left(s^{\prime}\right)_{0}=Z^{\prime}$ and the corresponding hyperplane $V^{\prime} \subset W$. The canonical map yields the exact triple

$$
0 \rightarrow V^{\prime} \otimes O_{X} \rightarrow E(Z, C) \rightarrow I_{Z^{\prime}}(H) \rightarrow 0
$$

and this shows that $E(Z, C) \cong E\left(Z^{\prime}, C\right)$, since $\operatorname{dim} \operatorname{Ext}\left(I_{Z^{\prime}}(H), O_{X}\right)=$ 1. 
Next we show the converse. The rank two bundles $E(Z, C) \cong E\left(Z^{\prime}\right.$, $\left.C^{\prime}\right) \cong E$ has two non-proportional sections $s$ and $s^{\prime}$ with zeros,

$$
(s)_{0}=Z,\left(s^{\prime}\right)_{0}=Z^{\prime} .
$$

The restriction of the canonical map $H^{0}(E) \otimes O_{X} \rightarrow E$ to the linear span $\left\langle s, s^{\prime}\right\rangle$ yields the following exact triple

$$
\begin{gathered}
0 \rightarrow\left\langle s, s^{\prime}\right\rangle \otimes O_{X} \rightarrow E \rightarrow L \rightarrow 0, \\
\operatorname{Supp} L=C=C^{\prime} \in|H|,
\end{gathered}
$$

since $h^{0}\left(I_{Z}(H)\right)=h^{0}\left(I_{Z^{\prime}}(H)\right)=1$. Then $L \cong O_{C}(Z) \cong O_{C}\left(Z^{\prime}\right)$.

So, this induces a map from $N^{\prime}=\left\{(L, C) \mid L \in \operatorname{Pic}^{c_{2}}(C), h^{0}(C, L)=\right.$ 2, $C$ a smooth curve in $|H|, h^{0}\left(I_{Z}(H)\right)=1$, where $Z$ is a zero section of $L\}$ to $M$. This is injective and the image is in $M_{0}^{0}$ as an open and dense subset in the closure $M^{\prime}$ in $M$ as was shown in theorem I. So, this completes the first part of the theorem.

For the second part we use the same method as in the previous theorem to conclude that $E$ corresponding to a singular point is $\pi_{*} L$ with $L^{2}=0$. So, $\operatorname{dim} M=\operatorname{dim} N$ must be $2 c_{2}-3>0$. This implies that for a generic curve $C \in|H|, \operatorname{dim} W_{c_{2}}^{1}(C)=\rho\left(c_{2}+1,1, c_{2}\right)=c_{2}-3$.

REMARK 1 . We do not know whether or not $M$ is irreducible, even though we believe it.

REMARK 2. The corresponding component in the moduli space of stable bundles on $\bar{X}$ is $M_{\bar{X}}^{0}\left(2, \pi^{*} H, \pi^{*} c_{2}\right)$. (See chapter I.) Then $M_{0}^{0}$ is mapped to $M_{\bar{X}}^{0}$ by $\pi^{*}$. The image of the bundle $E(Z, C) \in g(N)$ by $\pi^{*}$ is in the branch locus if and only if $O_{C}(Z)$ is a theta characteristic on $C\left(O_{C}(Z) \cong O_{C}\left(K_{C}-Z\right)\right)$. We expect that the intersection of the image of the moduli space by pull back map with the fixed locus of involution in [21] is of $\operatorname{dim} \frac{1}{2}\left(\operatorname{dim} M_{\bar{X}}+1\right)$.

REMARK 3. In theorem II, we assumed that $H^{2} \geq 6$, so that $c_{2} \geq 3$. However, $M$ is non-empty only if $H^{2} \geq 2\left(c_{2} \geq 1\right)$. In the case $H^{2}=2$, $c_{2}=1$, this corresponds to the case of the exceptional bundle. For $H^{2}=4, c_{2}=2$ and $H^{2}=6, c_{2}=3$, we will explain as examples.

EXAMPLE 1. $\left(H^{2}=4, c_{2}=2\right)$ If $|H|$ is non-hyperelliptic, then $M$ is just the locus of hyperelliptic curves in $|H|=\mathbf{P}^{2}$, since to each hyperelliptic curve $C$ we can associate a bundle uniquely determined by the hyperelliptic system on $C$. The image of $M$ by $\pi^{*}$ is one of the two elliptic curves in $M_{\bar{X}}$ which determine the double covering of $M_{\bar{X}}$ over 
$\mathbf{P}^{2}$. The moduli space of bundles with determinant $H+K$ are mapped to the other elliptic curve by $\pi^{*}$.

If $|H|$ is hyperelliptic, then $M$ is birational to some moduli space of bundles with $c_{1}^{2}=0$ and $c_{2}=1$. (See the remark 3 in chapter II.)

EXAMPLE 2. $\left(H^{2}=6, c_{2}=3\right)$ Every curve of genus 4 is trigonal. It is known ([19]) that a non-hyperelliptic curve of genus 4 has exactly two $g_{3}^{1}$ 's, $D$ and $K-D$ or one $g_{3}^{1}, D(\sim K-D)$. So, if $|H|$ is not hyperelliptic, then $M$ is birational to a double covering of $\mathbf{P}^{3}=|H|$ branched over the locus of curves which has a theta characteristic of $g_{3}^{1}$. If $|H|$ is hyperelliptic, then it can be transformed to another moduli space of bundles with $c_{1}^{2}=2$ and $c_{2}=2$.

\section{Remarks}

(1) Qin's result ([25]) shows that the birational type of the moduli space of stable bundles of rank 2 on an Enriques surface is independent of the choice of an ample divisor. In the case $c_{1}=0$, the moduli space can be described in two different ways. The first one is to transform to the cases (A) or (B) we described in the previous two chapters. The second one is to follow the description of Friedman's result. (He assumed that every fiber is irreducible, so that this corresponds to an unnodal Enriques surface.)

(2) In both theorems, we assumed that $X$ is unnodal. However, for a non-ample divisor $H$ on a nodal Enriques surface, our method does not work automatically. There might be a transformation to another moduli space of bundles whose determinant is ample with possibly different rank using exceptional bundles of even rank ([23]).

\section{References}

[1] C. Banica, M. Putinar, and G. Schumacher, Variation der globalen Ext in Deformationen kompakter komplexer Räume, Math. Ann. 250 (1980), no. 2, 135-155.

[2] W. Barth, Moduli of vector bundles on the projective plane, Invent. Math. 42 (1977), 63-91.

[3] W. Barth, C. Peters, and A. Van de Ven, Compact Complex Surfaces, SpringerVerlag, 1984.

[4] U. N. Bhosle, Nets of quadrics and vector bundles on a double plane, Math. Z. 192 (1986), no. 1, 29-43.

[5] E. Brosius, Rank 2 vector bundles on a ruled surface I, II, Math. Ann. 265 (1983), 155-168, Math. Ann. 266 (1983), 199-214. 
[6] F. Cossec and I. Dolgachev, Enrique Surfaces I, Progress in Mathematics, 76. Birkhaeuser Boston, Inc., Boston, MA (1989), Enrique Surfaces II, to appear.

[7] I. Dolgachev and I. Reider, On rank 2 vector bundles with $c_{1}^{2}=10, c_{2}=3$ on Enriques surfaces, LNM 1479 (1991).

[8] S. K. Donaldson and P. B. Kronheimer, The geometry of four-manifolds (1990), Oxford Mathematical Monographs, Oxford Science Publications, 1990.

[9] J.-M. Drezet and J. Le Potier, Fibrès stables et fibrès exceptionnels sur $P^{2}$, Ann. Sci. École Norm. Sup. 18 (1985), 193-243.

[10] R. Friedman, Rank two vector bundles over regular elliptic surfaces, Invent. Math. 96 (1989), no. 2, 283-332.

[11] R. Friedman and J. Morgan, On the diffeomorphism types of certain algebraic surfaces I, J. Diff. Geom. 27 (1988), no. 2, 297-369; II, J. Diff. Geom. 27 (1988), no. 3, 371-398.

[12] K. Hulek, Stable rank 2 vector bundles on $P^{2}$ with $c_{1}$ odd, Math. Ann. 242 (1979), no. 3, 241-266.

[13] H. Kim, Moduli spaces of stable vector bundles on Enriques surfaces, Nagoya Math. J. 150 (1998), 85-94.

[14] _ Moduli spaces of bundles mod Picard groups on some elliptic surfaces, Bull. Korean Math. Soc. 35 (1998), no. 1, 119-125.

[15] S. Mukai, Symplectic structure of the moduli space of sheaves on an abelian or K3 surface, Invent. Math. 77 (1984), no. 1, 101-116.

$[16] \ldots$, On the moduli space of bundles on $K 3$ surfaces $I$, Vector bundles on algebraic varieties (Bombay, 1984), 341-413, Tat Inst. Fund. Res. Stud. Math., 11, Bombay, 1987.

[17] C. Okonek, M. Schneider, and H. Spindler, Vector bundles on complex projective spaces, Progress in Math. Vol 3, Bikhaeuser, Boston, 1980.

[18] C. Okonek and A. Van de Ven, Stable bundles and differentiable structures on certain elliptic surfaces, Invent. Math. 86 (1986), no. 2, 357-370.

[19] S. Ramanan, Ample divisors on abelian surfaces, Proc. London Math. Soc. (3) 51 (1985), no. 2, 231-245.

[20] F. Takemoto, Stable vector bundles on algebraic surfaces II, Nagoya Math. J. Vol. 52 (1973), 173-195.

[21] A. N. Tyurin, Special 0 -cycles on a polarized surface of type $K 3$, Math. Ussr-Izv. 30 (1988), no. 1, 123-143.

[22] _ Cycles, curves and vector bundles on an algebraic surface, Duke Math. J. 54 (1987), no. 1, 1-26.

[23] __ Symplectic structures on the moduli surfaces of vector bundles on algebraic surfaces with $p_{g}>0$, Math. Ussr-Izv. 33 (1989), no. 1, 139-177.

[24] Z. Qin, Moduli spaces of stable rank-2 bundles on ruled surfaces, Invent. Math. 110 (1992), 615-626.

[25] — Chamber structures of algebraic surfaces with Kodaira dimension zero and moduli spaces of stable rank two bundles, Math. Z. 207 (1991), no. 1, 121136.

[26] A. Verra, On Enriques surface as a fourfold cover of $P^{2}$, Math. Ann. 266 (1983), no. 2, 241-250. 
Topology and Geometry Research Center Kyungpook National University

Taegu 702-701, Korea

E-mail: hikim@gauss.knu.ac.kr 